\section{Summary points}

In international clinical trials, scientists and sponsors in developed nations need to establish partnerships with stakeholders in developing countries

Partners should include researchers, government organisations, community leaders, and people who might be eligible for the trial

Partnerships must start during the planning stages of trials

Investigators and sponsors should take specific steps to promote partnerships

advisory board, and use partnerships to strengthen informed consent, help characterise the appropriate standard of care for the control group, and seek post-trial access to interventions. Review committees should include individuals who have experience with clinical trials in developing countries and understand the complexity of partnerships in this setting.

Partnerships should be continually strengthened-Just as researchers need to strive to improve recruitment and follow up during a clinical trial, they should also continually try to strengthen partnerships through quality improvement. Specific measures might include educational and outreach programmes to community groups, training for members of advisory boards about the scientific and ethical aspects of clinical trials, as well as attention to the dynamics of research team meetings.

Research team meetings need to promote partnershipsMeetings designed to build and sustain collaboration often subvert these goals because of cultural misunderstandings. Patterns of deference to authority based on age, experience, status, and sex may frustrate efforts to elicit feedback and discussion. As a result, collaborative meetings may actually contribute to or sustain a sense of disempowerment. Those who chair meetings must develop the skills to conduct project meetings in ways that ensure everyone speaks and is heard. For instance, chairs might need to explicitly invite people from developing countries to give their views, ask junior people to speak before more senior people have stated their position, or ask participants about their views in private as well as in meetings. Finally, chairs need to elicit the range of perspectives in communities-for example, by asking advisory board members what other perspectives are important in their communities.

\section{Conclusions}

By inclination and training, researchers may want to focus on the technical aspects of designing protocols and analysing data. However, researchers conducting clinical trials in developing countries have ethical obligations beyond those falling on researchers working in the developed world. ${ }^{4}$ No single profession, team, or country has a monopoly on wisdom. Establishing partnerships will assure procedural fairness and promote the ethical conduct of clinical trials in a world characterised by grave inequities.

Contributors and sources: BL was a member of the National Bioethics Advisory Committee, serves on a data and safety monitoring board for a clinical trial of preventing perinatal HIV transmission in Africa, has advised investigators on several international clinical trials funded by the National Institutes of Health, and has taught in China about ethical issues in research. $\mathrm{He}$ is codirector of the Policy and Ethics Core at the Center for AIDS Prevention Studies at the University of California San Francisco. RB has studied ethical challenges posed by the AIDS epidemic since the early 1980s. He has served on several Institute of Medicine committees that addressed these challenges and written and edited books on the subject. Members of the ethics working group participated in discussions of the topic and provided feedback and suggestions on drafts of manuscripts. Sources of information for this article include HIV Prevention Trials Network researchers, staff, and community advisory board members, reports on research in developing countries, and PubMed searches with the key words human experimentation, community, and third party consent. Funding: The HIV Prevention Trials Network is sponsored by the National Institute of Allergy and Infectious Diseases, National Institute of Child Health and Human Development, National Institute on Drug Abuse, National Institute of Mental Health, and Office of AIDS Research, of the National Institutes of Health, US Department of Health and Human Services (through a Cooperative Agreement to Family Health International, U01-AI-46749). The views in this article do not necessarily represent the views of the National Institutes of Health or the HIV Prevention Trials Network.

Competing interests: The authors receive research funding from the National Institutes of Health.

1 World Medical Association. Declaration of Helsinki: ethical principles for medical research involving human subjects. www.wma.net/e/policy/ b3.htm (accessed 10 Jun 2003).

2 National Bioethics Advisory Commission. Ethical and policy issues in international research: clinical trials in developing countries. Bethesda: international research: clinical trials in develop
National Bioethics Advisory Commission, 2001.

3 Council for International Organizations of Medical Sciences. InterCouncil for International Organizations of Medical Sciences. Inter-
national ethical guidelines for biomedical research involving human subjects, revised draft. Geneva: CIOMS, 2002. www.cioms.ch/ frame_guidelines_nov_2002.htm (accessed 10 Jun 2003).

4 Nuffield Council on Bioethics. The ethics of research related to healthcare in developing countries. London: Nuffield Council on Bioethics, 2002.

5 HIV Prevention Trials Network. Ethical guidance for research. www. hptn.org/ResearchEthics/HPTN_Ethics_Guidance.htm\&\#10;(accessed 13 June 2003).

6 Centers for Disease Control and Prevention. Building community partnerships in research:recommendations and strategies. Atlanta: CDCP, 1998.

7 Weijer C, Emanuel EJ. Protecting communities in biomedical research. Science 2000;289:1142-4.

8 Strauss RP, Sengupta S, Quinn SC, Goeppinger J, Spaulding C, Kegeles $\mathrm{SM}$, et al. The role of community advisory boards: involving communities in the informed consent process. Am J Public Health 2001;91:1938-43.

9 Sharp RR, Foster MW. Involving study populations in the review of genetic research.J Law Med Ethics 2000;28:41-51.

10 Juengst ET. Commentary: what "community review" can and cannot do. J Law Med Ethics 2000;28:52-4, 3.

11 Reilly PR. Rethinking risks to human subjects in genetic research. Am J Hum Genet 1998;63:682-5.

(Accepted 2 June 2003)

\section{Endpiece}

\section{A choice}

When you reach your 60s, you have to decide whether you're going to be a sot or an ascetic. In other words, if you want to go on working after you're 60 , some degree of asceticism is necessary.

$$
\text { Malcolm Muggeridge (1903-90), }
$$
British journalist

Fred Charatan,

retired geriatric physician, Florida 